\title{
Semantic Web Service Composition
}

Riina Henriksson

Helsinki November 26, 2006

UNIVERSITY OF HELSINKI

Department of Computer Science 


\section{Abstract}

Flexible and dynamic interoperation of autonomous information systems often requires automated or semiautomated combination of several highly distributed and heterogeneous web services. Web service composition aims to accelerate rapid application development and service reuse as well as to provide access to a variety of complex services. There are several approaches developed for the web service composition. This paper describes the overall process of designing composite services and the semantic web service composition approach.

The goal of semantic web service composition is to provide machine-readable descriptions of web services which will be used for automated discovery, composition, enactment and monitoring of these services. OWL-S (former DAML-S) is an ontology language for describing web services in terms of their inputs, outputs, preconditions, effects and their process model[HuS05]. OWL-S is still under development. First version of DAML-S was released in May 2001 and the latest version of OWL-S was pre-released in March 2006. Practical applicability of OWL-S was investigated by several research groups. Even though some problematic aspects of OWL-S were pointed out during applicability studies, OWL-S demonstrates its potential concerning the integration of proprietary data on the basis of ontologies. 


\section{Table of Contents}

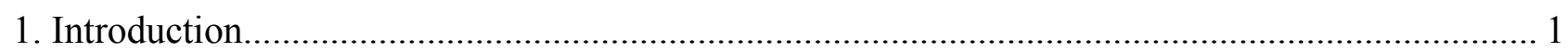

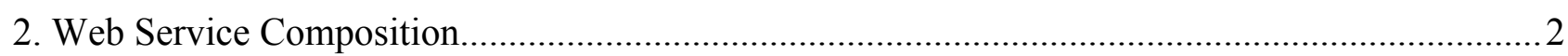

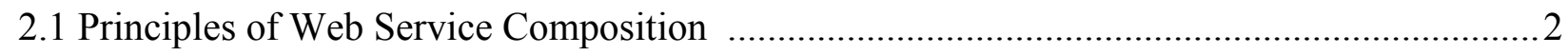

2.2 Web Service Composition Approaches........................................................................ 3

2.3 Web Service Composition Requirements.......................................................................... 4

3. Semantic Web Service Composition.......................................................................................... 5

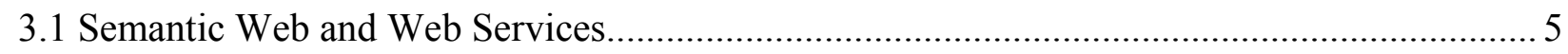

3.2 OWL-S: An Upper Ontology for Services......................................................................... 5

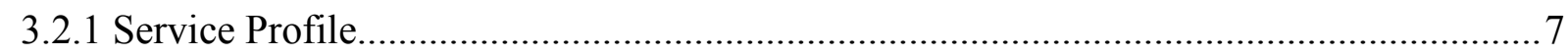

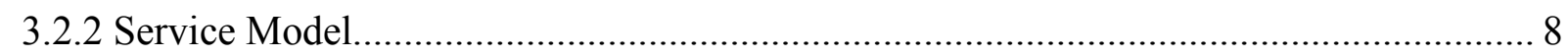

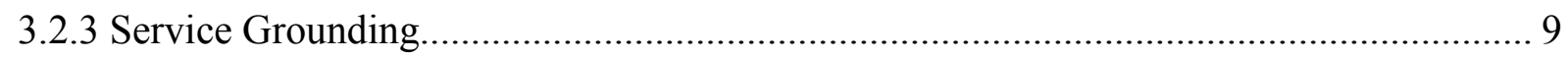

3.3 Automatic Composition of Semantic Web Services........................................................ 10

4. Evaluation of practical applicability of the current OWL-S specification.................................. 12

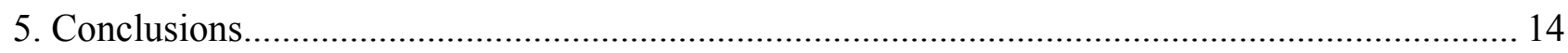

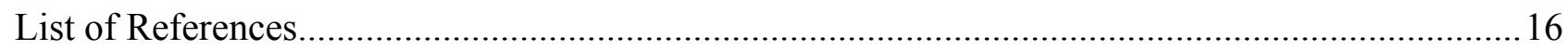





\section{Introduction}

The Web Service paradigm aims to enable dynamic and flexible interoperation of autonomous information systems. Available pre-existing web services can be composed to work together in order to achieve a goal specified by the client. Several approaches are developed in order to specify what is the right way to model web services and their compositions. This paper is aimed to provide a review on semantic web service composition approach.

OWL-S (previously known as DAML-S) is an ontology language for declaring and describing services. This semantic web service approach aims to provide an ontology that allows software agents to discover, execute and compose web services automatically [BLW04]. OWL-S is layered on the Ontology Web Language (OWL) which is a markup language for publishing and sharing data using ontologies on the Internet. Even though OWL-S is still under development, this approach aspires to become an industry standard and attracts a lot of interest.

This paper is structured as follows: Section 2 discusses principles of web service composition. Section 3 describes the OWL-S approach. Section 4 gives an overview on strengths and weaknesses in current OWL-S specification. Section 5 outlines the conclusions. 


\section{Web Service Composition}

\subsection{Principles of Web Service Composition}

In the world of service-oriented computing (SOC) applications are build by combining available web services in order to satisfy a client request. Service composition aims to accelerate rapid application development and service reuse as well as to provide access to a variety of complex services [MiM04].

The overall process of designing composite services includes the following key elements described in figure 1. In order to accomplish the goal, activities need to be discovered and orchestrated. The orchestration should be constrained by the information sharing [HuS05].

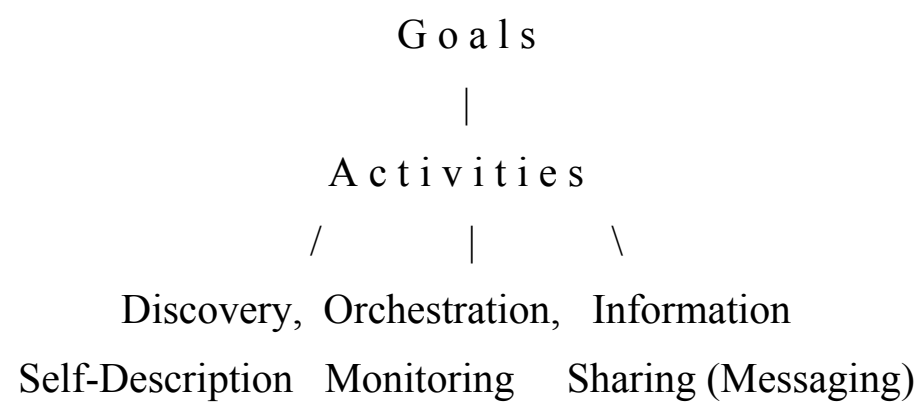

\section{Figure 1: Anatomy of Web Service Composition [HuS05]}

The composition process can be divided into two phases. Composition synthesis is a process of synthesizing a composite web service which results into a specification of how to coordinate available web services to make this composition work. This specification can be produced automatically or manually. Orchestration is a process of coordinating web services according to a given specification. This process includes monitoring control and data flow among the involved web services as well as taking care of correct execution of the composite service [Ber03].

Composition synthesis is quite challenging especially when synthesis is performed automatically. Another great challenge of service composition is validation of the composite service correctness: the goal should be achieved, there should be no deadlocks, termination should be correct etc. 


\subsection{Web Service Composition Approaches}

Several approaches to web service composition are proposed by research groups and organizations. These approaches range from those aspiring to become industry standards (BPEL and OWL-S) and more abstract methods [MiM04].

Business Process Execution Language for Web Services (BPEL) supports process-oriented service composition. In BPEL the result of composition is called process, participation services are partners and message exchange is called an activity. A process consists of a set of activities [MiM04]. Among design goals associated with BPEL is to define business processes using an XML based language and to define a set of web service orchestration concepts to be used by external and internal views of a business process [Wik06].

Semantic web service approach aims to provide an ontology that allows software agents to discover, execute and compose web services automatically [BLW04]. OWL-S defines an upper ontology for services which consists of a service profile for advertising and discovering services, a process model which gives a detailed description of a service's operation and a service grounding which provides details on how to interoperate with a service via message exchange. OWL-S is discussed in more detail in chapter 3.

In the Web Component approach services are treated as components where the composite-logic information is encapsulated inside a class definition. A public interface of such web component can be published and used for discovery and reuse. This approach supports sequential and parallel composition constructs.

In the Algebraic Service Composition approaches services are modeled as mobile processes. This approach is based on $\pi$-calculus theory and aims to provide simple descriptions which can be efficiently verified. 
Services can be modeled also as Petri Nets. A Petri net is a directed, connected and bipartite graph where nodes represent places and transitions and tokens occupy places. In this approach each service has a petri net where transitions are assigned to methods and places to states [MiM04]. A petri net describes service behavior and has one port for input and one port for output. When a petri net definition for each service is completed, a composition operator performs the composition. As a result of composition process new service is created. An advantage of this approach is that petri nets can be used to prove the absence of deadlocks and correct termination.

In Conversation specification approach individual services (peers) communicate through asynchronous messages. Each individual service has a queue for incoming messages and there is a global "watcher" to keep track on messages. The conversation is a sequence of messages [Bul03]. This conversation among the peers models the global behavior of the service composition. In this approach services are modeled as Mealy machines which are finite-state machines with input and output. A finite state machine (FSM) or finite-state automata is a model of behavior composed of states, transitions and actions [Wik06]. In this approach the correctness of a web service composition (properties such as data consistency, deadlock avoidance, and business-constraint satisfaction) can be verified by checking correctness inside a workflow specification [MiM04].

\subsection{Web Service Composition Requirements}

Service composition approaches described above are quite different, however there are some common requirements for all composition approaches. When some available pre-existing web services are combined to work together in order to achieve a specified goal the correctness of this composition should be validated. A service composition should also guarantee connectivity and scalability as well as nonfunctional Quality of Service requirements such as timeliness, security and dependability [MiM04].

A great challenge for people working with service compositions is that there is no standardization available yet. In several research papers it is said that application development is done in a ad hoc way, without standard framework or libraries; additionally there is a lack of methodologies and tools. Thus there is still a lot of research needed in many aspects of service composition. 


\section{Semantic Web Service Composition}

\subsection{Semantic Web and Web Services}

Web has became an important media for individuals and organizations. However search of some relevant and reliable information on the web is rather a challenging process which is based on using suitable keywords. Furthermore a person using the keyword based information search can not fully rely on the search results generated by an automated search process but needs to know how and where information can be found. The same issue is valid for web services. A web service listed in UDDI (Universal Description, Discovery and Integration) registry can be located manually or automatically, however this service may be still not suitable for several reasons such as technical or geographical limitations, legal aspects, usage pre-conditions etc.; or simply because this service does not provide a desired solution.

The Semantic Web paradigm aims to provide possibility to access information and services by content as well as keywords. Web markup languages such as OWL enable to create ontologies for any domain. An ontology is an explicit and formal specification of a conceptualization [AnH04]. Semantic Web aims also to enable automatic location, selection, employment, composition and monitoring of web services [Bur06]. Such intelligent service provisioning can be implemented through semantic descriptions of services and requires a framework within which service descriptions can be made and shared.

\subsection{OWL-S: An Upper Ontology for Services}

OWL-S is an ontology for declaring and describing services which is developed as a collaborative effort of several researches and organizations. OWL-S is referred also as a language for describing services as it provides a standard vocabulary to create service descriptions. OWL-S is based on the Ontology Web Language (OWL) Recommendation produced by the Web-Ontology Working Group at the World Wide Web Consortium (W3C) [Bur06]. OWL is a markup language for publishing and sharing data using ontologies on the Internet [Wik06]. OWL-S is still evolving; the 
current version 1.1 was pre-released in March 2006. This chapter describes OWL-S in more detail based on specification provided by M. Burstein, et al. in [Bur06].

OWL-S has following three tasks: automatic web service discovery, automatic web service invocation as well as automatic web service composition and interoperation.

\section{Automatic web service discovery}

When searching for a web service a client usually knows what service capabilities he is looking for and what are the constraints which should be taking into consideration. Such information can be specified as computer-interpretable semantic markup at the service web sites and thus located automatically by an ontology-enhanced search engine or a service registry. Additionally OWL-S enables declarative advertisements of service properties and capabilities.

\section{Automatic web service invocation}

Execution of a web service can be implemented as a collection of remote procedure calls. In order to execute web service automatically, a software agent needs to understand what input is required to invoke the service and what information will be returned. In comparison to a pre-programmed solution, only a declarative description of this service is given here. OWL-S specifies a declarative, computer-interpretable API that includes the semantics of the arguments for remote procedure calls and the semantics for returned messages.

\section{Automatic web service composition and interoperation}

OWL-S provides a language for description of service compositions and data flow interactions. Additionally in OWL-S can be specified information about prerequisites and consequences of usage of individual services.

In OWL-S a service has three essential types of knowledge as shown in Figure 2. ServiceProfile specifies what the service provides for clients. ServiceModel descries how the service is used. ServiceGrounding provides details how to interact with the service. 


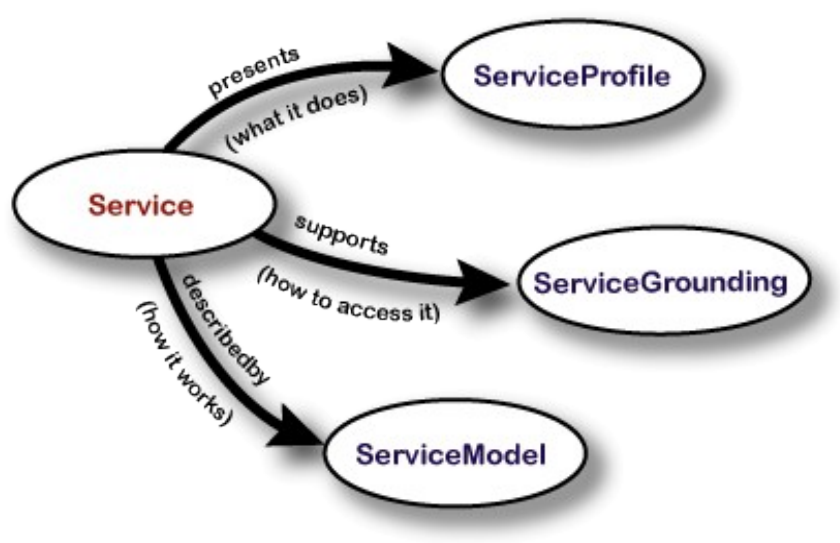

Figure 2: Top level of the service ontology [Bur06]

This ontology for services specifies only two cardinality constraints: a service can be described by at most one service model, and a grounding must be associated with exactly one service.

\subsubsection{Service Profile}

Service Profiles can be used by service providers to publish web services. These Service Profiles are referred as advertisements. Service Profiles can be also used by service requesters to describe a web service to be searched for. During discovery process this request is compared with available advertisements and this way the suitable service is found.

Information stored in Service Profiles aims to provide a concise description of the service and can be divided into three categories: semi-structured information about service and service provider, a functional description and additional non-functional parameters such as a category and a quality rating to describe features of this service ([BLW04], [Bur06]). The semi-structured information is meant for information which is not relevant for semantic service discovery but can be valuable for human users. The functional description of the service includes inputs and preconditions required to execute this service as well as outputs and effects generated by the service execution.

Once service has been selected the Service Profile is useless as the client will use the Process Model to interact with the service. Service Profile and Process Model are two different representations of a service and they have different roles. Profile specifies the intended purpose of the service meaning that only those functionalities of service are advertised which the organization wants to provide and some functionalities may be hided. Service profile claims to be an universal declarative description of a service and thus can be used in any type of registry ([BLW04], [Bur06]). 


\subsubsection{Service Model}

In OWL-S service is modeled as a process, which is viewed as a specification of the ways a client may interact with a service [Bur06]. This approach is based on work in several fields such as distributed systems, process modeling, workflow technology, modeling verb semantics and event structure as well as work in AI on standardizations of planning languages and modeling complex actions.

A service model can be composed using different types of processes and control constructs. OWL-S defines three classes of process (see Figure 3). An atomic process is used to describe a service that expects one input message and returns one message in response. Such process is directly invocable and has no subprocesses. A simple process is an abstraction mechanism which can be used either to provide a view of some atomic process or a simplified representation of a composite process which may be useful for planning and reasoning. A composite process is a composition of other composite or atomic processes and maintains some state information. This process type provides a specification of how inputs are accepted by particular subprocesses and how its various outputs are produced by particular subprocesses. With other words, the composite process represents a behavior the client can perform by sending and receiving a series of messages.

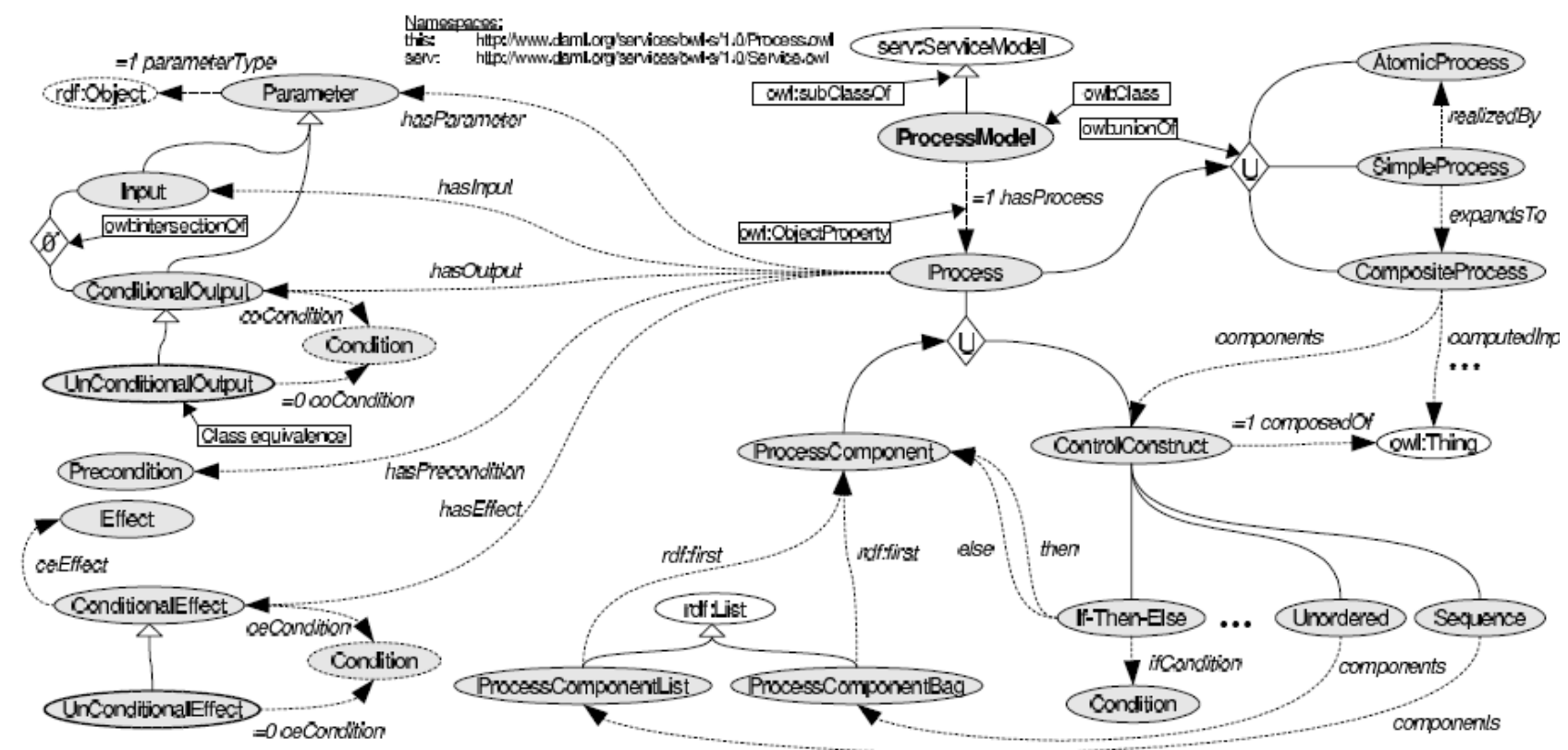


Figure 3: OWL-S process ontology [BLW04]

A composite process can be considered as a tree where nonterminal nodes are labeled with control constructs and leaves are invocations of other processes. The following control constructs are available in OWL-S:

\begin{tabular}{|l|l|}
\hline $\begin{array}{c}\text { OWL-S control } \\
\text { construct }\end{array}$ & \multicolumn{1}{c|}{ Description } \\
\hline Sequence & The component processes are executed in a specified order. \\
\hline Split & $\begin{array}{l}\text { The component processes of a Split process are executed concurrently. Split } \\
\text { completes when all component processes have been scheduled for execution. }\end{array}$ \\
\hline Split + Join & $\begin{array}{l}\text { The component processes of a Split+Join process are executed concurrently } \\
\text { and with barrier synchronization. Slit+Join completes when all of its } \\
\text { components are completed. }\end{array}$ \\
\hline Any-Order & $\begin{array}{l}\text { The component processes are executed in some unspecified order and not } \\
\text { concurrently. Execution and completion of all components is required. }\end{array}$ \\
\hline Choice & $\begin{array}{l}\text { A single control construct is called from a given bag of control constructs and } \\
\text { executed. }\end{array}$ \\
\hline If-Then-Else & $\begin{array}{l}\text { This control construct has properties to specify if-condition as well as then and } \\
\text { else and follows the following semantics: "test if-condition, if true do then, if } \\
\text { false do else" }\end{array}$ \\
\hline Iterate & $\begin{array}{l}\text { This control construct provides a possibility to specify conditions for initiation, } \\
\text { termination and maintenance with while-condition or until-condition. }\end{array}$ \\
\hline $\begin{array}{l}\text { Repeat-While and } \\
\text { Repeat-Until }\end{array}$ & $\begin{array}{l}\text { Both control constructs follow the familiar programming language } \\
\text { conventions. Repeat-While may never act, Repeat-Until always acts at least } \\
\text { once. }\end{array}$ \\
\hline
\end{tabular}

\subsubsection{Service Grounding}

Service profile and process model represent an abstract specifications of web service characteristics. The grounding of a service specifies how to communicate with a concrete web service by binding abstract IOs of atomic processes to concrete message formats. This grounding mechanism reuses the extensive work done in Web Service Description Language (WSDL). OWL-S provides an ontology for grounding process descriptions to WSDL which is based on three correspondences between OWL-S and WSDL (see Figure 4) 


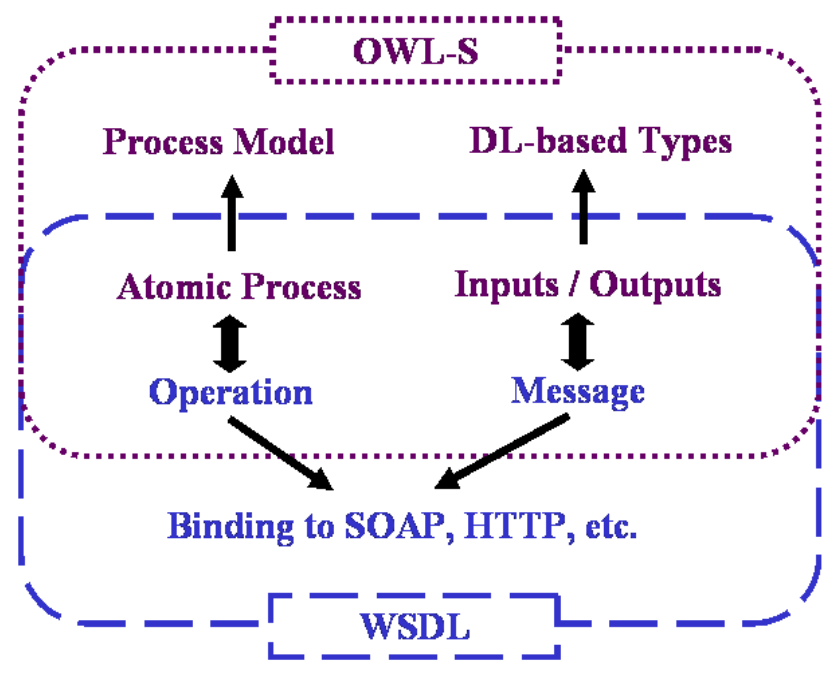

Figure 4: Mapping between OWL-S and WSDL [Bur06]

Firstly, An atomic process in OWL-S corresponds to a WSDL (version 1.1) operation. Secondly, OWL-S inputs correspond to the parts of an input message of a WSDL's operation, and OWL-S outputs correspond to WSDL's output message. Thirdly, the types of the inputs and outputs of an OWL-S atomic process correspond to the concept of abstract type in WSDL. The following steps are required to construct an OWL-S/WSDL grounding: first the messages and operations by which an atomic process may be accessed should be identified in WSDL, then the correspondences should be specified.

\subsection{Automatic Composition of Semantic Web Services}

OWL-S provides possibility to describe content and capabilities of web services with semantical markup which can be used as a base for automatic web service composition. Many research groups have been choosing OWL-S when studying issues related to automatic web service compositions and implementing practical use cases ([BLW04], [Bur06], [NaM02]). In this chapter one of the approaches to composition developed in context of OWL-S is discussed.

In the approach proposed by S. Narayanan and S. McIlraith OWL-S descriptions are mapped first into a Situation Calculus and then encoded into a Petri Net formalism. The goal of a web service 
composition is specified here in terms of an overall effect to be achieved starting from initial state [HuS05].

The situation calculus language used in this approach is a first-order logical language for representing dynamically changing worlds where all the changes are results of actions performed by some agent [NaM02]. Situations are sequences of actions evolving from initial situation. Atomic processes in OWL-S are actions in the situation calculus. The conditional effects are represented in the situation calculus as positive and negative effect axioms and outputs are treated as knowledge effects. Preconditions for an atomic process are represented as well-formed formula and inputs are threated as knowledge preconditions.

The Petri Nets approach, which was briefly described in chapter 2.2, provides distributed operational semantics of processes. After the situation calculus is translated into a Petri Net representation it is possible to accomplish both offline analysis tasks such as web service composition and online execution tasks such as deadlock determination resource satisfaction and quantitative performance analysis.

This research group has constructed an execution semantics for OWL-S based on Petri Nets. Additionally an interpreter which reads OWL-S files and outputs a network representation was implemented. The constructed network can be visualized using KarmaSIM simulation environment. Furthermore a variety of analysis techniques such as reachability analysis, deadlock detection, etc. can be applied to verify the correctness of composition. 


\section{Evaluation of practical applicability of the current OWL-S specification}

Even though OWL-S is still under development, it attracts a lot of interest among researches. Several publications on OWL-S ([HuS05], [BLW04], [Mik04], [NaM02]) conclude that one of the strengths of OWL-S is the possibility to provide formal semantics for describing web services. Additionally as stated in [SCZ04] OWL-S scores highly in expressiveness over other available languages and has well-defined semantics in description logic. Nevertheless many researchers are not convinced that the current OWL-S specification is sufficient to meat the goal of automatic web service discovery, composition and monitoring.

Since OWL-S is layered on top of OWL it is influenced by disadvantages common for OWL. The biggest disadvantage in term of web service composition is the lack of variables within OWL [BLW04]. Without a possibility to reference particular parameters for describing the flow of data with help of variables, inputs and outputs of atomic processes can not be related to each other. In some cases the OWL-S specification conflicts in some cases with OWL language semantics. Some publications ([BLW04], [Mik04]) suggest that OWL-S is not a suitable formalism for representing procedural knowledge. In publication by P. Mika, et al. [Mik04] it is suggested to align OWL-S to DOLCE, a Descriptive Ontology for Linguistic and Cognitive Engineering which is a part of Foundational Ontology Library (WFOL). This alignment could gain some improvements in design and provide wider scope for service descriptions [Mik04].

OWL-S is partitioning web services into profiles, process models and grounding which is valuable with respect to different phases of finding and execution of web services [BLW04]. However web services in many cases are only a part of the overall service process and thus service descriptions cross the boundary between an information system and the real world [Mik04]. From this point of view web service representation proposed by OWL-S can be considered being too narrow to describe independent views on the world by the various actors involved. In the current version of OWL-S it is unclear how to distinguish objects and events within information system and the real world objects and events external to this system. Additionally in OWL-S approach a service can be related to exactly one service model, meaning that there can not be any alternative service models for one service. 
There are many other pitfalls of OWL-S reported in research publications. One area which is not covered in OWL-S is the service composition runtime behavior. Mapping input and output parameters and specifying preconditions and effects helps to describe initial and finite state of a service but this is not sufficient to specify and verify runtime behavior of service composition [SCZ04]. Validation and exception handling are other aspects which needs to be improved in OWL$\mathrm{S}$ [SCZ04]. Researchers have proposed methods for transferring OWL-S descriptions to Prolog and Petri-net based notation in order to improve verification issues [MiM04]. However, the extent to which correctness of composition is verified varies.

A practical application of OWL-S is challenging as there are no tools available to support the modeling and verification of OWL-S descriptions on a high semantic level. In order to create OWLS descriptions a comprehensive knowledge of OWL and OWL-S as well as WSDL, SOAP and $\mathrm{XSLT}$ is required [BLW04].

It seems that OWL-S is gaining lots of critic, however this critic is mostly constructive. There are several suggestions how to improve the weaknesses found in the current specification of OWL-S which will probably contribute to the further development of this approach. 


\section{Conclusions}

Research efforts in area of web service composition are mainly concerned with two challenges in this field: automated service synthesis and verification of the correctness in the web service composition. There are many approaches and languages developed for the service composition, but non of them is fully resolving these challenges yet.

Semantic Web Service approach aims to enable automatic locations, selection, employment, composition and monitoring of web services. OWL-S is an ontology for declaring and describing services which is developed as a collaborative effort of several researches and organizations. OWL-S is partitioning web services into profiles, process models and groundings which is valuable with respect to different phases of finding and execution of web services. A possibility to describe content and capabilities of web services with semantical markup provides a base for automatic web service composition.

OWL-S has been receiving some critics as revealed from the literature review. One remarkable disadvantage in term of web service composition is the lack of variables, which limits possibilities to describe data flows in a service composition. Additionally web service representation proposed by OWL-S is considered by some researchers being too narrow to describe independent views on the world by the various actors involved. Furthermore several researchers have found that such issues as runtime behavior of service composition, exception handling and verification of composition correctness are not covered sufficiently in the current specification of OWL-S

Nevertheless many research groups have been using OWL-S service descriptions as a base for implementing web service compositions. As a result several approaches and workarounds have been proposed. One promising approach is proposed by S. Narayanan and S. McIlraith, where OWL-S descriptions are mapped first into a Situation Calculus and then encoded into a Petri Net formalism. As a result a Petri Nert representation provides possibility to accomplish both offline analysis tasks such as web service composition and online execution tasks such as deadlock determination resource satisfaction and quantitative performance analysis.

Semantic web services is a promising approach, but there is still some work to be done especially in 
the area of compositional specification and verification of services in this domain. 


\section{List of References}

[AnH04] G. Antoniou, F. v. Harmelen. A Semantic Web Primer. The MIT Press, 2004

[Ber03] D. Berardi, D. Calvanese, G. De Giacomo, M. Lenzerini, M. Mecella. Automatic Composition of E-Services That Export Their Behavior. ICSOC 2003

[BLW04] S.Balzer, T.Liebig, M.Wagner. Pitfalls of OWL-S - A Practical Semantic Web Use Case. ICSOC'04. November 15-19, 2004

[Bul03] T.Bultan, X. Fu, R. Hull, J. Su. A New Approach to Design and Analysis of EService Composition. WWW2003, May 20-24, 2003, Budapest, Hungary

[Bur06] M. Burstein, et al. OWL-S: Semantic Markup for Web Services, available at http://www.ai.sri.com/daml/services/owl-s/1.2/overview/

[HuS05] R. Hull, J. Su. Tools for Composite Web Services: A Short Overview. SIGMOD record, Vol. 34, No. 2, June 2005

[Mik04] P.Mika, A.Gangemi, D.Oberle, M.Sabou. Foundations for Service Ontologies:

Aligning OWL-S to DOLCE. WWW2004, May 17-22, 2004

[MiM04] N. Milanovic, M. Malek. Current Solutions for Web Service Composition. IEEE Computer Society, November-December 2004

[NaM02] S. Narayanan, S. McIlraith. Simulation, Verification and Automated Composition of Web Services. WWW2002, May 7-11, 2002, Honolulu, Hawaii, USA

[SCZ04] M. Solanki, A. Cau, H. Zedan. Augmenting Semantic Web Service Description with Compositional Specification. WWW2004, May 17-22, 2004

[Wik06] Wikipedia. available at www.wikipedia.com

[W3C02] W3C Web Service Activity home page. http://www.w3.org/2002/ws/ 